\title{
Common Usability Problems and Solutions for Science Gateways
}

\author{
Paul Parsons \\ Polytechnic Institute \\ Purdue University \\ West Lafayette, IN, USA \\ parsonsp@purdue.edu \\ Yu-Chuan Chen \\ Polytechnic Institute \\ Purdue University \\ West Lafayette, IN, USA \\ chen3056@purdue.edu
}

Brandon Dentler

Polytechnic Institute

Purdue University

West Lafayette, IN, USA

bdentler@purdue.edu

\author{
Yu-Shen Ho \\ San Diego Supercomputer Center \\ University of California, San Diego \\ La Jolla, CA, USA \\ connieho1222@gmail.com
}

Kaitlin Groothuis

Polytechnic Institute

Purdue University

West Lafayette, IN, USA

kgroothu@purdue.edu

\author{
Claire Stirm \\ San Diego Supercomputer Center \\ University of California, San Diego \\ La Jolla, CA, USA \\ stirm@sdsc.edu
}

\author{
Sandra Gesing \\ Center for Research Computing \\ University of Notre Dame \\ Notre Dame, IN, USA \\ sandra.gesing@nd.edu
}

Mike Zentner

San Diego Supercomputer Center

University of California, San Diego

La Jolla, CA, USA

mzentner@ucsd.edu

\begin{abstract}
Science gateways require a wide range of considerations to make them successful and sustainable. One important consideration is usability. Gateway creators typically lack usability expertise and do not know how to avoid common usability problems. The Science Gateways Community Institute (SGCI) has provided numerous workshops, webinars, and consulting services to the community to improve gateway usability. Based on more than 30 in-depth usability evaluations conducted over the past three years, we identify a small set of common usability problems that gateways have. We characterize these problems and present some possible solutions. Additionally, we show examples of problems and solutions from gateways we have worked with. This paper can help gateways creators identify common usability problems along with solutions that are easy for non-experts to implement.
\end{abstract}

Index Terms-usability, science gateways, cyberinfrastructure

\section{INTRODUCTION}

Science gateways are digital interfaces that provide access to shared resources such as software, data repositories, computational services, and educational materials. They serve as knowledge platforms for a range of community membersincluding researchers, developers, educators, and studentsacross a wide variety of disciplines. While gateways are often intended to serve the needs of a specific scientific community, building an effective gateway requires far more than disciplinary expertise-e.g., gateways with long-term sustainability tend to have good business and marketing plans, clear goals and mission statements, appropriate cybersecurity initiatives, and a strong focus on usability [1], [2].
The NSF-funded Science Gateways Community Institute (SGCI) serves the gateways community by providing consulting services addressing a range of topics needed to create successful gateways [3], [25]. Topics include full-stack development, research computing, cybersecurity, business and marketing, finance and budgeting, project management, and usability. Of the different consulting services offered, usability is the most popular, making up $43 \%$ of all active and completed engagements. Through SGCI's usability consulting, gateway creators receive usability evaluations and high-level strategy advice from graduate and undergraduate students who are part of an innovative program in User Experience (UX) Design at Purdue University [4], [18].

The importance of usability was identified in the early days of science gateways scholarship; in 2010, Wilkins-Diehr and Lawrence [6] held a focus group of fifteen participants with gateways experience to discuss factors that influence gateway sustainability. During this focus group, numerous participants attributed the success of their gateways to good usability, among other things. In more recent years, usability evaluation has been increasingly desired and valued by gateways teamsapproximately $65 \%$ of administrators and application creators reported an anticipated need for usability help with their projects in a large survey of the community [1]. Responding to this need, SGCI provides one-to-three-month usability engagements for gateway teams, doing a deep evaluation of the gateway and providing suggestions for redesigns.

While an appreciation for the importance of usability is 
apparent within the gateways community, not much has been written that aims to provide usability guidance specifically to gateway creators. Although there is a large body of literature on usability in a general sense, gateways creators often do not know where to look or which of the literature is applicable to their context. In this paper, we report on a small number of usability issues that we commonly encounter in our consulting work with gateways creators. We describe these issues and propose solutions that are actionable for non experts.

\section{Context And Method}

SGCI's usability team has conducted more than 30 in-depth usability engagements with gateways teams over the past 3 years. We have worked with teams across a wide variety of domains and disciplines, including hurricane modeling, intercultural learning, nanotechnology, chemistry education, library science, ocean science, and others. Engagements with gateways teams typically last 2-3 months and involve a series of deliverables based on the evaluation and design methods that we use. Common usability evaluation methods that we use include interviews and surveys with users and creators, usability testing with users [7], [8], competitive analyses, heuristic evaluations [9], [10], and cognitive walkthroughs [11]. We also provide high-level design suggestions using a variety of ideation and prototyping methods common among user-centered designers.

To identify a small set of the most common usability problems that we have encountered in our consulting work, we performed a lightweight content analysis on our own reports that have been generated for each of the consulting engagements. When we perform usability evaluations, we document the process and findings, and integrate them into a final report. This final report indicates the significant problems and our suggestions for addressing them.

By doing this analysis, our goal is to identify a small set of issues that are common and can be remedied by gateways creators without special training or outside help. It is important to note that the issues presented here are not the only common or important ones that gateways creators may encounter, but we believe that they are universal enough that most gateways creators would benefit from being aware of them and knowing something about their possible solutions.

\section{Common Usability Problems}

Our analysis identified three usability problems that are common in the majority of our gateways evaluations: (1) users are confused about where to look; (2) users are overwhelmed by text; and (3) users are lost in the application. We articulate the issues from the user's perspective, as it encourages a shift in thinking from a developer's view to the user's view, which is a fundamental pillar of user-centered design. With some basic knowledge, we believe these issues are easy to detect and remedy for the majority of gateways. We describe these and provide some possible solutions in the subsections below.

\section{A. Users are confused about where to look}

When users first encounter a gateway, their eyes look for something to initially anchor onto, in order to start making sense of what they are seeing. Salient features naturally draw attention, and they can be used to tell users where to look. Visual characteristics such as size, color, alignment, and contrast can be used to create a visual hierarchy, guiding the order in which users look at items on a page. However, when these characteristics are not used, or not used appropriately, users will not know where to look or how to make sense of the new environment. In such cases, users get frustrated and may not utilize the gateway optimally, or may not use it at all.

Solution: Ensure visual hierarchy. Make use of features such as size, color, contrast, proximity, and motion to create a visual hierarchy that will guide the user's attention in ways that are desired. For example, if a link is very important, make the text large and put it in a colored box to make users see it first. Consider which elements users should see first, second, and third, and make use of the aforementioned visual characteristics to encourage such viewing patterns. Aside from this bottom-up approach to attention, users' goals also drive their attention [13], [14], so it is important to consider the visual hierarchy with respect to users goals.

Figure 1 shows the landing page for the nanoHUB gateway [5], where a visual hierarchy is employed to guide the user's attention. Here we can see the tagline using large, bolded text with a high color contrast, creating a strong probability that users will look there first. We also see the four categories of content contained within large boxes, also using contrast and spacing to increase the probability that users will look there before other areas. Finally, we see the sub-heading and the menu items using smaller, unbolded text with a lower contrast, increasing the probability that users will look there after the other items.

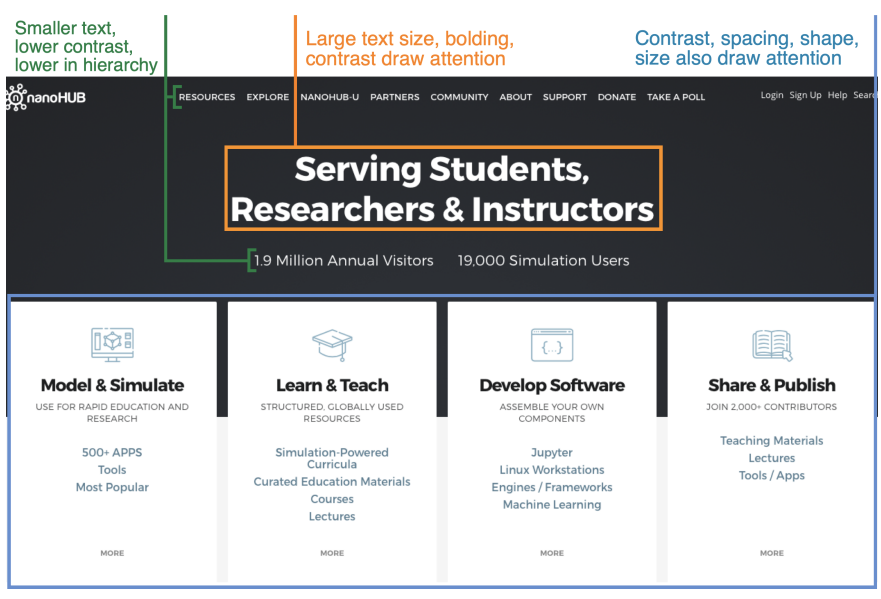

Fig. 1. The nanoHUB gateway [5] using visual hierarchy to guide attention.

\section{B. Users are overwhelmed by text}

Gateways are typically created by researchers and developers who are used to dealing with text-heavy documents, such 
as technical reports and scientific papers. In such documents, detailed writing is important, and readers expect ideas to be developed over a series of sentences and paragraphs. Readers of websites and apps, however, do not usually share the same expectations. Instead, they expect text to be scannable, such that they can quickly move around and identify important pieces of information without having to read long sentences and paragraphs. When there is a mismatch between users' expectations of content and what they are given, they can become overwhelmed. We regularly see gateways with far too much text that quickly overwhelms users. Gateways creators often think they need much text to tell users what the gateways is about; however, if users really want such detailed information, they will make the effort to read it on another part of the gateway (e.g., an 'about' page).

Solution: Write scannable text. It is important for gateways creators to know the differences between writing technical documents and writing for the web. Web users typically scan text and rarely read word-by-word. Text can be made more scannable by emphasizing keywords (see visual hierarchy above), using bulleted lists, choosing meaningful headings, and keeping paragraphs short and limited to a single idea only [23]. Less text can ironically lead to users spending more time reading. For example, in one relatively simple usability study [15], the average time people spent on the features page of a website increased about $30 \%$ after the page was redesigned to significantly reduce the amount of text.

Figure 2 shows a portion of the homepage for the QC Archive gateway [12], where scannable text is used to not overwhelm users. Text is succint, well-aligned, and accompanied by visual items. Users can quickly scan the page without having to read linearly top-to-bottom.
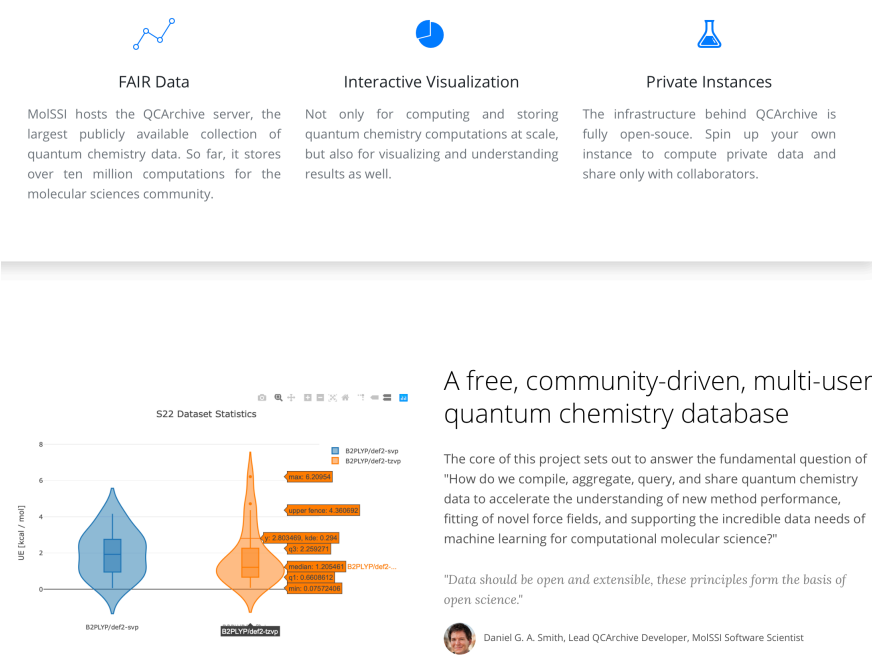

Fig. 2. QC Archive gateway [12] using scannable text that does not overwhelm users.

\section{Users are lost in the application}

With most gateways, users need to navigate through multiple pages or screens while performing their tasks. In such cases, it is easy for users-especially novices-to feel lost and not know 'where they are' within the application. For example, when browsing a site, users may feel confused about which page they are on, which page they came from, or how to get back to a previous page they were on. Ideally, users should always be able to answer the following questions from anywhere within a gateway application: Where am I? Where have I been? Where can I go? In our evaluations, we often see cases where these questions are not easily answerable by users, leading to frustration and a loss of efficiency.

Solution: Provide navigational cues. To help users understand where they are within an application, clear visual cues can be used to answer the three navigational questions mentioned above. For example, visual 'breadcrumbs' are a well-known interface design pattern for enhancing navigability by conveying where users are within an application's hierarchy [16]. Other methods include changing the background color of a menu item to indicate the current page, providing navigational overviews for long pages, and providing clear categories when there is a significant amount of content.

Figures 3 and 4 show examples of gateways using common patterns for indicating to a user where they are within a site. Figure 3 shows a common 'breadcrumb' technique, where the hierarchical location within the site is shown, with '/ indicating a level within the hierarchy. This technique is useful when there is a deep hierarchy to the site. Figure 4 shows a common technique for indicating which page the user is on, where a colored line is used as a visual indicator. This technique is useful for showing which main section of the site a user is in, but does not show hierarchical depth. Both techniques could be combined to show the main sub-page and depth to the user.

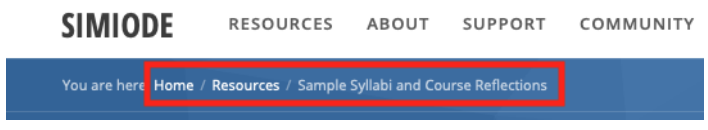

Fig. 3. SIMIODE gateway [22] using the 'breadcrumb' technique to indicate hierarchical location in the site.

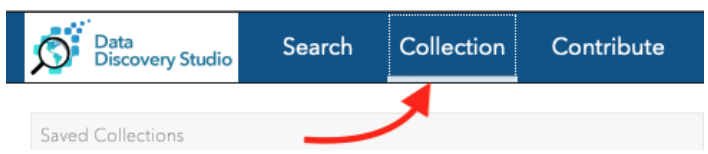

Fig. 4. DataDiscoveryStudio.org gateway [21] using a colored underline to indicate the current page.

Table I summarizes the three usability problems that we have commonly encountered in our consulting work with science gateways creators. A single possible solution is suggested for each one based on the discussion and examples above.

\section{REDESIGN EXAMPLES}

Here we briefly discuss two examples of problems and solutions from usability evaluations that we have done with two different gateways. Figure 5 shows a screen from the 
TABLE I

COMMON USABILITY PROBLEMS AND SOME POSSIBLE SOLUTIONS

\begin{tabular}{|l|l|}
\hline Common Usability Problems & Possible Solutions \\
\hline Users are confused about where to look & Ensure visual hierarchy \\
Users are overwhelmed by text & Write scannable text \\
Users are lost in the application & Provide navigational cues \\
\hline
\end{tabular}

DataDiscoveryStudio.org gateway [21] before the usability evaluation. As can be seen, while there is some visual hierarchy being employed, there could be more; there is a significant amount of text without much structure; and there are no navigational supports within the page except for scrolling. Figure 6 shows the same page after a redesign. The title is simplified, removing some text. Although the main body of text needed to stay, it was categorized with navigational supports on the left side, which also serve as bulleted text that is easily scannable.

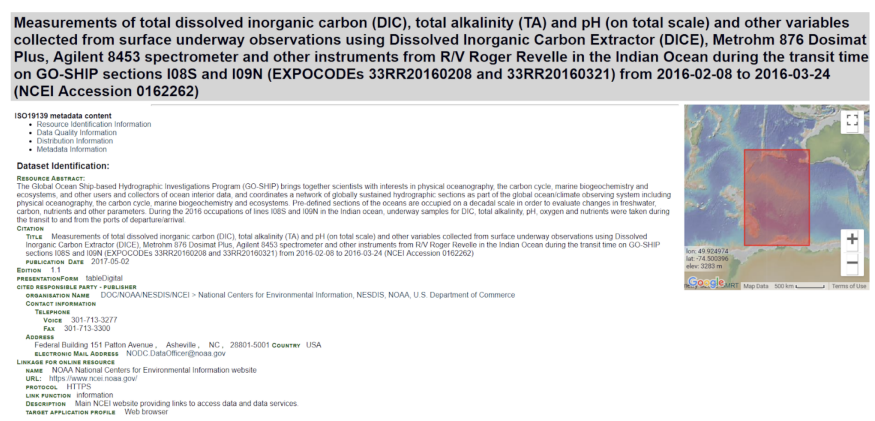

Fig. 5. Original page from the DataDiscoveryStudio.org gateway [21].

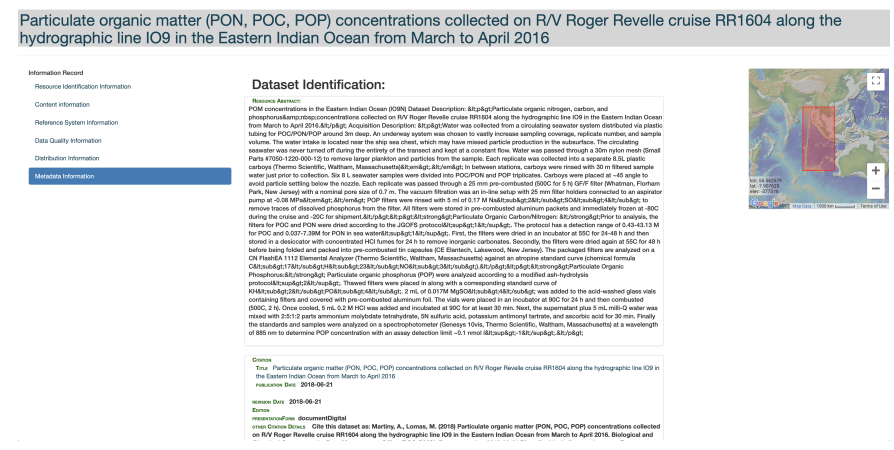

Fig. 6. Improved version of the page from Figure 5, with better visual hierarchy, scannable text, and navigational cues.

Figure 7 shows a screen from the SIMIODE gateway before the usability evaluation [22]. While there is some visual hierarchy conveyed with colored text and headings, there is not enough to clearly guide attention; there is also a large amount of text that is not easily scannable. Figure 8 shows a redesign of the content within the red box. Large icons were used to attract visual attention; short labels were used for scannability; a title was provided for hierarchy; and the overall text was simplified.

These two examples-while simple-illustrate small changes that can be made to gateways to make noticeable improvements to their usability.

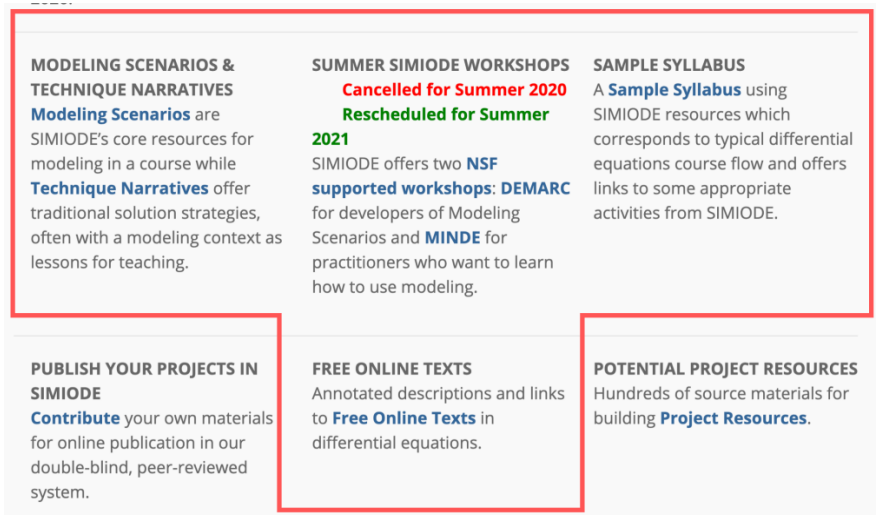

Fig. 7. Original page from the SIMIODE gateway [22].

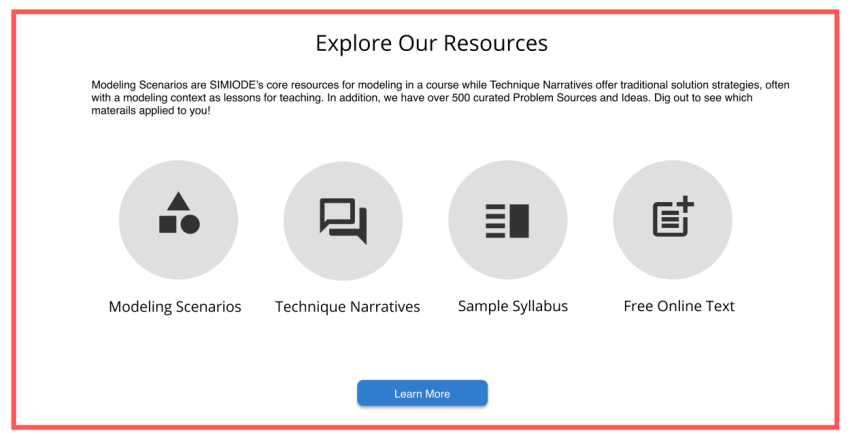

Fig. 8. Improved version of the page from Figure 7, with better visual hierarchy, scannable text, and navigational cues.

\section{Gateway-Specific Challenges}

Aside from general usability challenges, there have sometimes been challenges specific to gateways that we have observed throughout our consulting work. We briefly discuss three common ones below.

\section{A. No usability expertise on gateway team}

Sometimes gateway teams are concerned about not having an internal usability expert and not having funding to hire one. Our response has always been that doing basic usability work is easier than many people anticipate. There are numerous resources for running simple usability tests with users, including various websites [17] and books [7], [24]. Redesigns like the ones shown above are simple to do and do not require trained usability specialists or web designers to implement.

\section{B. Gateways are using 3rd party software}

Gateways often incorporate third-party software, such as Jupyter Notebooks [19] and Apache Airavata [20], into the user's workflow. Sometimes these software do not allow for easy customization of the user interface. If this is the case, the best option is to ensure the entry point into the third-party software is well designed. Make sure users know why they are making a context shift into the software, how they get there in terms of their navigation and task flow, how they get back, and how the third-party software fits into the overall goals 
of the gateway. Following these suggestions can significantly improve the usability of a gateway even though the third-party software is not modified in any direct way.

\section{Encountering survivorship bias}

A common way for gateway teams to receive feedback on usability is to reach out to existing users. While this can be a good practice, there is a danger of sampling only current users. Current users can be thought of as 'survivors', i.e., the ones who have made it through and stuck with the gateway. While their perspectives can be valuable, focusing on them alone ignores all the potential users who may have tried the gateway and not stuck with it-perhaps it was too confusing or difficult to use. Existing users have also become familiar with the gateway, and are more likely to view it positively due to that familiarity. One of the easiest ways to overcome this bias is to conduct usability tests with non-users. Such users can be recruited externally (e.g., see [15]), which can be time consuming and expensive, or they can simply be colleagues or students that are available for quick usability testing. Asking a colleague to conduct a simple usability test for 20 minutes can reveal usability issues that longtime users may not be able to surface nearly as easily.

\section{SUMMARY}

Science gateways require careful attention to usability if they are to be successful and sustainable in the long term. Most gateway teams do not have internal usability expertise, and may not have funds to hire outside experts. Based on our experience conducting more than 30 in-depth usability engagements with science gateways, we have identified three common usability problems and some solutions. These problems are easy for non-experts to identify, and the solutions are similarly easy to implement. By sharing these, we hope that gateway creators are able to easily improve the usability of their gateways and provide better experiences for their users.

\section{ACKNOWLEDGMENTS}

This work has been funded by NSF grant 1547611 . We would like to thank all of the gateways that have worked with us and participated in our events. We would also like to thank the many students from Purdue University that have contributed to the usability consulting over the years.

\section{REFERENCES}

[1] K. A. Lawrence et al., "Science gateways today and tomorrow: positive perspectives of nearly 5000 members of the research community," Concurrency and Computation: Practice and Experience, vol. 27, no. 16, pp. 4252-4268, 2015, doi: 10.1002/cpe.3526.

[2] S. Gesing, "Science Gateways in HPC: Usability Meets Efficiency and Effectiveness," in Modeling and Simulation in HPC and Cloud Systems, J. Kołodziej, F. Pop, C. Dobre, Eds. Springer, 2018.

[3] S. Gesing et al., "Science gateways: the long road to the birth of an institute," in Proceedings of the 50th Hawaii International Conference on System Sciences, 2017, doi: 10.24251/HICSS.2017.755.

[4] M. Vorvoreanu, C. M. Gray, P. Parsons, and N. Rasche, "Advancing UX education: A model for integrated studio pedagogy," in Proceedings of the 2017 SIGCHI Conference on Human Factors in Computing Systems (CHI '17), 2017, pp. 1441-1446.
[5] G. Klimeck, M. McLennan, S. P. Brophy, G. B. Adams III, and M. S. Lundstrom, "nanohub. org: Advancing education and research in nanotechnology," Computing in Science Engineering, 10(5), 17-23, 2008.

[6] N. Wilkins-Diehr and K. A. Lawrence, "Opening science gateways to future success: The challenges of gateway sustainability," 2010 Gateway Computing Environments Workshop (GCE), New Orleans, LA, 2010, pp. 1-10, doi: 10.1109/GCE.2010.5676121.

[7] J. S. Dumas and J. Redish, "Introducing usability testing," in A Practical Guide to Usability Testing, 2nd ed. Oregon, USA: Intellect Books, 1999.

[8] J. C. Bastien, "Usability testing: a review of some methodological and technical aspects of the method," International journal of medical informatics, vol. 79, issue 4, pp. e18-e23, April 2010, doi: 10.1016/j.ijmedinf.2008.12.004

[9] J. Nielsen and R. Molich, "Heuristic evaluation of user interfaces, " in Proceedings of the SIGCHI Conference on Human Factors in Computing Systems (CHI '90), New York, pp. 249-256, doi: 10.1145/97243.97281.

[10] J. Nielsen, "Usability inspection methods," in Conference companion on Human factors in computing systems, 1994, pp. 413-414.

[11] P. G. Polson, C. Lewis, J. Rieman, and C. Wharton, "Cognitive walkthroughs: a method for theory-based evaluation of user interfaces," International Journal of man-machine studies, vol. 36, issue 5, pp. 741773, 1992, doi: 10.1016/0020-7373(92)90039-N.

[12] D. G. Smith, D. Altarawy, L. A. Burns, M. Welborn, L. N. Naden, L. Ward, ... and T. D. Crawford, "The MolSSI QCArchive project: An open-source platform to compute, organize, and share quantum chemistry data," Wiley Interdisciplinary Reviews: Computational Molecular Science, e1491, 2020.

[13] S. Djamasbi, M. Siegel, T. Tullis, "Visual Hierarchy and Viewing Behavior: An Eye Tracking Study," in International Conference on HumanComputer Interaction, pp. 331-340, Springer, Berlin, Heidelberg, 2011, doi: 10.1007/978-3-642-21602-2_36.

[14] J. Nielsen and K. Pernice, "Eyetracking web usability," New Riders, 2010.

[15] G. Kogan, Usability Testing is Easier Than You Think, May 7, 2014. Accessed on: May 17, 2020. [Online]. Available: https://www.gkogan.co/blog/usability-testing-case-study/

[16] B. L. Rogers and B. Chaparro, "Breadcrumb navigation: Further investigation of usage," Usability News, vol. 5, issue 2, pp. 1-7, 2003.

[17] K. Moran, Usability Testing 101, Neilsen Norman Group, December 1, 2019. Accessed on May 17, 2020. [Online]. Available: https://www.nngroup.com/articles/usability-testing-101/

[18] C. M. Gray, P. Parsons, A. L. Toombs, N. Rasche, and M. Vorvoreanu, "Designing an Aesthetic Learner Experience: UX, Instructional Design, and Design Pedagogy," International Journal of Designs for Learning, 11(1), 2020, pp. 41-58.

[19] T. Kluyver et al., "Jupyter Notebooks-a publishing format for reproducible computational workflows," in ELPUB, May 2016, pp. 87-90.

[20] S. Marru et al., "Apache airavata: a framework for distributed applications and computational workflows," Proceedings of the 2011 ACM workshop on Gateway computing environments, 2011, pp. 21-28.

[21] I. Zaslavsky, D. Valentine, S. M. Richard, O. Meier, B. PeuckerEhrenbrink, K. I. Stocks, "Beyond Search: Metadata Enhancement and In-Depth Data Exploration in EarthCube Data Discovery Studio," AGUFM, 2019, pp. IN53A-04.

[22] B. Winkel, Reasons for SIMIODE - White Paper, SIMIODE, Nov. 4, 2016. Accessed on: May 18, 2020. [Online]. Available: https://simiode.org/resources/3028.

[23] "How Users Read on the Web", Nielsen Norman Group, Accessed May 17, 2020. [Online]. Available: https://www.nngroup.com/articles/howusers-read-on-the-web

[24] S. Krug, "Rocket surgery made easy: The do-it-yourself guide to finding and fixing usability problems", New Riders, 2009.

[25] P. Parsons, S. Gesing, C. Stirm, and M. Zentner, "SGCI Incubator and its Role in Workforce Development: Lessons Learned from Training, Consultancy, and Building a Community of Community-Builders for Science Gateways," in Proceedings of the Practice and Experience in Advanced Research Computing Conference (PEARC '20), 2020, pp. 491-494, doi: 10.1145/3311790.3400850. 